\title{
Article
}

\section{Fixed Points Theorems for Unsaturated and Saturated Classes of Contractive Mappings in Banach Spaces}

\author{
Vasile Berinde ${ }^{1,2, *,+} \mathbb{1}$ and Mădălina Păcurar $3,+(\mathbb{D}$ \\ 1 Department of Mathematics and Computer Science, North University Center at Baia Mare, \\ Technical University of Cluj-Napoca, Victoriei 76, 430122 Baia Mare, Romania \\ 2 Academy of Romanian Scientists, Ilfov Str. no. 3, 050045 Bucharest, Romania \\ 3 Department of Economics and Bussiness Administration in German Language, Faculty of Economics and \\ Bussiness Administration, "Babeş-Bolyai" University of Cluj-Napoca, T. Mihali 58-60, 400591 Cluj-Napoca, \\ Romania; madalina.pacurar@econ.ubbcluj.ro \\ * Correspondence: vasile.berinde@mi.utcluj.ro \\ + These authors contributed equally to this work.
}

check for updates

Citation: Berinde, V.; Păcurar, M. Fixed Points Theorems for Unsaturated and Saturated Classes of Contractive Mappings in Banach Spaces. Symmetry 2021, 13, 713. https://doi.org/10.3390/sym13040713

Academic Editors: Isaac Pesenson and Sergei D. Odintsov

Received: 30 March 2021

Accepted: 14 April 2021

Published: 18 April 2021

Publisher's Note: MDPI stays neutral with regard to jurisdictional claims in published maps and institutional affiliations.

Copyright: (C) 2021 by the authors. Licensee MDPI, Basel, Switzerland. This article is an open access article distributed under the terms and conditions of the Creative Commons Attribution (CC BY) license (https:// creativecommons.org/licenses/by/ $4.0 /)$.

\begin{abstract}
Based on the technique of enriching contractive type mappings, a technique that has been used successfully in some recent papers, we introduce the concept of a saturated class of contractive mappings. We show that, from this perspective, the contractive type mappings in the metric fixed point theory can be separated into two distinct classes, unsaturated and saturated, and that, for any unsaturated class of mappings, the technique of enriching contractive type mappings provides genuine new fixed-point results. We illustrate the concept by surveying some significant fixed-point results obtained recently for five remarkable unsaturated classes of contractive mappings. In the second part of the paper, we also identify two important classes of saturated contractive mappings, whose main feature is that they cannot be enlarged by enriching the contractive mappings.
\end{abstract}

Keywords: Banach space; enriched contraction; averaged operator; enriched Kannan mapping; enriched nonexpansive mapping; fixed point; iterative method; unsaturated class of contractive mappings; saturated class of contractive mappings

\section{Introduction}

Let $(X, d)$ be a metric space and let $T: X \rightarrow X$ be a self mapping. We denote the set of fixed points of $T$ by Fix $(T)$, i.e., Fix $(T)=\{a \in X: T a=a\}$ and define the $n$th iterate of $T$ as usual; that is, $T^{0}=I$ (identity map) and $T^{n}=T^{n-1} \circ T$, for $n \geq 1$.

The mapping $T: X \rightarrow X$ is called a Banach contraction if there exists a constant $c \in[0,1)$ such that

$$
d(T x, T y) \leq c \cdot d(x, y), \text { for all } x, y \in X .
$$

Recall that the mapping $T$ is said to be a Picard operator, see, for example, Rus [1], if $(i)$ Fix $(T)=\{p\}$ and $(i i) T^{n} x_{0} \rightarrow p$ as $n \rightarrow \infty$, for any $x_{0}$ in $X$.

The Banach contraction mapping principle, first introduced by Banach [2] in the setting of a Banach space and then extended by Caccioppoli [3] to the setting of a complete metric space, essentially states that, in a complete metric space $(X, d)$, any Banach contraction $T: X \rightarrow X$ is a Picard operator.

We note that in condition (1), two displacements appear out of the six possible displacements of two distinct points $x, y \in X$ :

$$
d(T x, T y), d(x, y), d(x, T x), d(y, T y), d(x, T y) \text { and } d(y, T x) .
$$

In the development of metric fixed-point theory, in order to obtain a metric fixed-point result for a mapping $T$, two were used, as in the case of the Banach contraction condition (1), with three or more displacements from the list (2), as we shall see in the following. 
One condition which uses three displacements from the list (2) was introduced in 1969 by Kannan [4,5] and is extremely important in fixed-point theory for many reasons. One reason is that, while any Banach contraction is continuous, Kannan mappings are generally not continuous, thus showing that Picard operators are not necessarily continuous.

A mapping $T: X \rightarrow X$ is called a Kannan mapping if there is a constant $a \in[0,1 / 2)$, such that

$$
d(T x, T y) \leq a(d(x, T x)+d(y, T y)), \text { for all } x, y \in X
$$

Theorem 1 ([4]). Let $(X, d)$ be a complete metric space and let $T: X \rightarrow X$ be a Kannan mapping. Then, $T$ is a Picard operator.

In 1972, Chatterjea [6] introduced the following dual of the Kannan contraction condition

$$
d(T x, T y) \leq b[d(x, T y)+d(y, T x)], \forall x, y \in X
$$

where $b \in[0,1 / 2)$ is a constant, for which a similar fixed point theorem to Theorem 1 holds.

It is known, see [7-9], that conditions (1), (3) and (4) are independent, that is, there are Banach contractions which are not Kannan mappings, and Kannan mappings which are not Banach contractions, etc. For more details and discussions on this topic, see $[7,8,10,11]$.

Based on the previous observation, in 1972, Zamfirescu [12] formulated a very interesting fixed-point theorem that involves all three conditions (1), (3), and (4).

Theorem 2 ([12]). Let $(X, d)$ be a complete metric space and $T: X \longrightarrow X$ a map for which the real numbers $a, b$ and $c$ exist, satisfying $0 \leq a<1,0<b, c<1 / 2$, such that, for each pair, $x, y$ in $X$, at least one of the following is true:

$$
\begin{aligned}
& \left(z_{1}\right) d(T x, T y) \leq \operatorname{ad}(x, y) ; \\
& \left(z_{2}\right) d(T x, T y) \leq b[d(x, T x)+d(y, T y)] ; \\
& \left(z_{3}\right) d(T x, T y) \leq c[d(x, T y)+d(y, T x)] .
\end{aligned}
$$

Then, $T$ is a Picard operator.

In 2004, Berinde [9] unified all previous classes of contractive mappings by means of the non symmetric contraction condition (5) and established an existence and approximation fixed-point result, while all the above-mentioned theorems establish the existence and uniqueness of the fixed point; see [13] for a recent survey on the fixed point theory of single-valued almost contractions.

Definition 1 ([9]). Let $(X, d)$ be a metric space. A mapping $T: X \rightarrow X$ is called a $(\delta, L)$-almost contraction if the constants $\delta \in(0,1)$ and $L \geq 0$ exist, such that

$$
d(T x, T y) \leq \delta \cdot d(x, y)+L d(y, T x), \quad \text { for all } x, y \in X
$$

Another general contraction conditions in this family, which involves all six displacements in the list (2) and, due to L. B. Ćirić, see [14], it also unifies all previous contractive mappings, except for the class of almost contractions (5).

A map $T: X \rightarrow X$ is called a Ćirić quasi contraction if there exists $h \in(0,1)$ such that, for all $x, y \in X$,

$$
d(T x, T y) \leq h \max \{d(x, y), d(x, T x), d(y, T y), d(x, T y), d(y, T x)\}
$$

It is important to note that (5) and (6) are independent classes of contractive mappings; see $[9,13]$. For the impressively rich literature on this topic, we refer to the monographs $[1,10,11,15,16]$, and the references therein.

On the other hand, in the recent papers [17-23], the authors used the technique of enrichment of contractive type mappings in order to generalize, in the setting of a Hilbert 
or a Banach space, well-known and important contractive type mapping classes from the metric fixed-point theory, satisfying symmetricand non symmetric metric conditions.

The technique of enriching contractive mappings has been suggested by the developments in fixed-point theory related to the concept of asymptotic regularity. This notion was formally introduced in 1966 by Browder and Petryshyn ([24], Definition 1, p. 572) in connection with the study of fixed points of nonexpansive mappings, but the same property was used in 1955 by Krasnoselskij [25] to prove that if $K$ is a compact convex subset of a uniformly convex Banach space and if $T: K \rightarrow K$ is nonexpansive, then, for any $x_{0} \in K$, the sequence

$$
x_{n+1}=\frac{1}{2}\left(x_{n}+T x_{n}\right), n \geq 0,
$$

converges to a fixed point of $T$.

In proving his result, Krasnoselskij used the fact that if $T$ is nonexpansive, which, in general, is not asymptotically regular, then the averaged mapping involved in (7), that is, $\frac{1}{2} I+\frac{1}{2} T$, is asymptotically regular. For the general averaged mapping

$$
T_{\lambda}:=(1-\lambda) I+\lambda T, \lambda \in(0,1),
$$

and in the setting of a Hilbert space, the corresponding result has been stated by Browder and Petryshyn ([26], Corollary to Theorem 5), while Ishikawa [27] proved the same property with no restriction on the geometry of the Banach space involved in 1976.

Therefore, the averaged operator $T_{\lambda}$ is enriching the class of nonexpansive mappings with respect to the asymptotic regularity. This fact suggested that one could similarly enrich the classes of contractive mappings in metrical fixed-point theory by imposing that $T_{\lambda}$, rather than $T$, satisfies a certain contractive condition.

In this way, the following mapping classes were introduced and studied: enriched contractions and enriched $\varphi$-contractions [20], enriched Kannan contractions [21], enriched Chatterjea mappings [22], enriched almost contractions [23], enriched nonexpansive mappings in Hilbert spaces [18], enriched nonexpansive mappings in Banach spaces [18], enriched strictly pseudocontractive operators in Hilbert spaces [17], semigroups of enriched nonexpansive mappings [28], etc.

We note that all the above-mentioned fixed-point results for enriched mappings were established in Banach or Hilbert spaces, which are metric spaces with generous geometrical properties. In order to extend those fixed-point results to the setting of a metric space $(X, d)$, one needs some additional geometric properties of the space $X$, related to usual sense of convexity for subsets of the Euclidian space, and expressed by the fact that, for any two distinct points $x$ and $y$ in $X$, there exists a third point $z$ in $X$ lying between $x$ and $y$.

Using an appropriate convexity structure in metric spaces, introduced by Takahashi [29], some of the above-mentioned results for enriched contractions, that is, those for enriched contractions and enriched $\varphi$-contractions [20], have subsequently been extended to the more general setting of convex metric spaces [21].

Moreover, after a close examination of the new fixed-point results, reported in [17-23], we noted that the technique of enriching contractive type mappings $T$ by means of the averaged operator $T_{\lambda}$ cannot effectively enlarge all classes of contractive mappings, as initially expected.

This observation suggested a new interesting concept, that of saturated class of contractive mappings with respect to the averaged operator $T_{\lambda}$, a notion which will be introduced in the next section.

We therefore analyse some well-known classes of contractive type mappings, which are either saturated or unsaturated.

For some of the above unsaturated classes of contractive mappings, we present various significant fixed-point results with appropriate illustrative examples, while, in the second part of the paper, we also identify two important classes of saturated contractive mappings, whose main feature is that they cannot be enlarged by means of the technique of enriching contractive mappings. 


\section{Unsaturated Classes of Contractive Mappings}

In this section, we present five classes of unsaturated contractive mappings: Banach contractions, Kannan contractions, Chatterjea contractions, almost contractions and nonexpansive mappings. For each class, after showing why it is unsaturated, we also indicate some relevant fixed-point results obtained by the technique of enriching the respective class.

First, we need to recall an important property that is fundamental in obtaining all fixed-point results for enriched contractive mappings reported in [17-20,22,23].

Let $T: C \rightarrow C$ be a self mapping of a convex subset $C$ of a linear space $X$. Then, for any $\lambda \in(0,1]$, the so-called averaged mapping (a term coined in [30]) $T_{\lambda}$ given by

$$
T_{\lambda} x=(1-\lambda) x+\lambda T x, \forall x \in C,
$$

has the property that

$$
\operatorname{Fix}\left(T_{\lambda}\right)=\operatorname{Fix}(T)
$$

Definition 2. Let $(X,\|\cdot\|)$ be a linear normed space and let $\mathcal{C}$ be a subset of the family of all self-mappings of $X$. A mapping $T: X \rightarrow X$ is said to be $\mathcal{C}$-enriched or enriched with respect to $\mathcal{C}$ if there exists $\lambda \in(0,1]$ such that $T_{\lambda} \in \mathcal{C}$.

Using $\mathcal{C}^{e}$, we denote the set of all enriched mappings with respect to $\mathcal{C}$.

Remark 1. From Definition 2, it immediately follows that $\mathcal{C} \subseteq \mathcal{C}^{e}$.

Definition 3. Let $X$ be a linear vector space and let $\mathcal{C}$ be a subset of the family of all self-mappings of $X$. If $\mathcal{C}=\mathcal{C}^{e}$, we say that $\mathcal{C}$ is a saturated class of mappings; otherwise, $\mathcal{C}$ is said to be unsaturated.

Note that $\mathcal{C}$ is unsaturated if and only if the inclusion $\mathcal{C} \subset \mathcal{C}^{e}$ is strict.

The main aim of this section is to present some important examples of unsaturated classes of contractive mappings, each of them with a relevant new fixed point result.

\subsection{Banach Contractions}

Let $(X, d)$ be a metric space and let $\mathcal{C}_{B}(X)$ denote the class of Banach contractions defined on $X$; that is, the class of all mappings which satisfy a contractive condition of the form (1) on $X$.

We further consider $(X,\|\cdot\|)$ as a linear normed space, and we consider the set of all mappings $T: X \rightarrow X$ which satisfy

$$
\|b(x-y)+T x-T y\| \leq \theta\|x-y\|, \forall x, y \in X,
$$

for some $b \in[0,+\infty)$ and $\theta \in[0, b+1)$.

If we take $\lambda=\frac{1}{b+1}$ and $c=\lambda \theta<1$ from (10), then we obtain that $T_{\lambda}$ satisfies (1); that is, $T_{\lambda} \in \mathcal{C}_{B}(X)$. According to Definition 2, this means that $T$ satisfying (10) is an enriched contraction (as introduced in [20], Definition 2.1), so we may denote, by $\mathcal{C}_{B}^{e}(X)$, the set of all self mappings of $X$ satisfying (10).

Obviously, $\mathcal{C}_{B}(X) \subseteq \mathcal{C}_{B}^{e}(X)$, since any Banach contraction satisfies (10) with $b=0$.

However, as the following example shows, the previous inclusion is strict, as not any enriched Banach contraction is a Banach contraction.

Example 1. Let $X=[0,1]$ be endowed with the usual norm and let $T: X \rightarrow X$ be defined by $T x=1-x$, for all $x \in[0,1]$. Then $T \notin \mathcal{C}_{B}(X)$ (just take $x=0, y=1$ in (1) to obtain the contradiction $1 \leq c<1$ ).

But $T \in \mathcal{C}_{B}^{e}(X)$ as $T_{\lambda} \in \mathcal{C}_{B}(X)$, for any $\lambda \in\left(0, \frac{1}{2}\right)$.

The above facts enable us to formulate the following result. 
Proposition 1. Let $(X,\|\cdot\|)$ be a Banach space. Then, $\mathcal{C}_{B}(X)$ is an unsaturated class of contractive mappings.

Proposition 1 now guarantees that the next theorem, the main result in [20], is a genuine generalization of the Banach contraction principle in the setting of Banach spaces.

Theorem 3 ([20], Theorem 2.4). Let $(X,\|\cdot\|)$ be a Banach space and $T: X \rightarrow X a(b, \theta)$-enriched contraction. Then

(i) Fix $(T)=\{p\}$, for some $p \in X$;

(ii) There exists $\lambda \in(0,1]$ such that the iterative method $\left\{x_{n}\right\}_{n=0}^{\infty}$, given by

$$
x_{n+1}=(1-\lambda) x_{n}+\lambda T x_{n}, n \geq 0,
$$

converges to $p$, for any $x_{0} \in X$;

(iii) The following estimate holds

$$
\left\|x_{n+i-1}-p\right\| \leq \frac{c^{i}}{1-c} \cdot\left\|x_{n}-x_{n-1}\right\|, \quad n=0,1,2, \ldots ; i=1,2, \ldots,
$$

where $c=\frac{\theta}{b+1}$.

\subsection{Kannan Contractions}

Let $(X, d)$ be a metric space and let $\mathcal{C}_{K}(X)$ denote the class of Kannan contractions defined on $X$, that is, the class of all mappings which satisfy a contractive condition of the form (3) on $X$.

We consider the set of all mappings $T: X \rightarrow X$ which satisfy

$$
\|k(x-y)+T x-T y\| \leq a(\|x-T x\|+\|y-T y\|), \text { for all } x, y \in X,
$$

for some $a \in[0,1 / 2)$ and $k \in[0, \infty)$, where $(X,\|\cdot\|)$ is a linear normed space.

If we take $\lambda=\frac{1}{k+1} \in(0,1]$, then, from (11), we obtain that $T_{\lambda} \in \mathcal{C}_{K}(X)$. According to Definition 2, this means that $T$ satisfying (11) is an enriched Kannan contraction (as was introduced in [21], Definition 2.1), so we may denote by $\mathcal{C}_{K}^{e}(X)$ the set of all self mappings of $X$ satisfying (11).

Obviously, any Kannan mapping satisfies (11) with $k=0$, which means that $\mathcal{C}_{K}(X) \subseteq \mathcal{C}_{K}^{e}(X)$.

However, as shown by the next example, the inclusion of $\mathcal{C}_{K}(X) \subset \mathcal{C}_{K}^{e}(X)$ is strict, as not every enriched Kannan contraction is a Kannan contraction.

Example 2. Let $X$ and $T$ be as in Example 1. Then-see Example 2.1 in [21] for details-for any $a \in[0,1 / 2)$ and $\lambda=\frac{1}{2-2 a}$, we have $T_{\lambda} \in \mathcal{C}_{K}(X)$.

However, $T$ is not a Kannan mapping. Indeed, if $T$ would be a Kannan mapping, that is, $T$ would satisfy (3), then there would exist $a \in[0,1 / 2)$ such that

$$
|x-y| \leq a \cdot(|2 x-1|+|2 y-1|), \text { for all } x, y \in[0,1],
$$

which, for $x=1 / 2$ and $y=1$, yields the contradiction $1 / 2 \leq a<1 / 2$.

So, $T \notin \mathcal{C}_{K}(X)$.

Now, based on the above facts, we can formulate the following result.

Proposition 2. Let $(X,\|\cdot, \cdot\|)$ be a Banach space. Then, $\mathcal{C}_{K}(X)$ is an unsaturated class of contractive mappings. 
Therefore, Proposition 2 guaranties that the next theorem, which is the main result in [21], is a genuine generalization of the Kannan fixed point theorem in the setting of Banach spaces.

Theorem 4 ([21], Theorem 2.1). Let $(X,\|\cdot\|)$ be a Banach space and $T: X \rightarrow X a(k, a)$-enriched Kannan mapping. Then

(i) Fix $(T)=\{p\}$, for some $p \in X$;

(ii) There exists $\lambda \in(0,1]$ such that the iterative method $\left\{x_{n}\right\}_{n=0}^{\infty}$, given by

$$
x_{n+1}=(1-\lambda) x_{n}+\lambda T x_{n}, n \geq 0,
$$

converges to $p$, for any $x_{0} \in X$;

(iii) The following estimate holds

$$
\left\|x_{n+i-1}-p\right\| \leq \frac{\delta^{i}}{1-\delta} \cdot\left\|x_{n}-x_{n-1}\right\|, \quad n=0,1,2, \ldots ; i=1,2, \ldots
$$

where $\delta=\frac{a}{1-a}$.

\subsection{Chatterjea Contractions}

Let $(X, d)$ be a metric space and let $\mathcal{C}_{C}(X)$ denote the class of Chatterjea contractions defined on $X$, that is, the class of all mappings which satisfy a contractive condition of the form (4) on $X$.

Now consider the set of all mappings $T: X \rightarrow X$ which satisfy

$$
\begin{gathered}
\|k(x-y)+T x-T y\| \leq b[\|(k+1)(x-y)+y-T y\|+ \\
+\|(k+1)(y-x)+x-T x\|], \forall x, y \in X
\end{gathered}
$$

for some $a \in[0,1 / 2)$ and $k \in[0, \infty)$, where $(X,\|\cdot\|)$ is a linear normed space.

If we take $\lambda=\frac{1}{k+1}$ in (12), then we have $0<\lambda \leq 1$ and

$$
\left\|T_{\lambda} x-T_{\lambda} y\right\| \leq b\left[\left\|x-T_{\lambda} y\right\|+\left\|y-T_{\lambda} x\right\|\right], \forall x, y \in X,
$$

with $b \in[0,1 / 2)$, which shows that $T_{\lambda} \in \mathcal{C}_{C}(X)$. According to Definition 2, this means that $T$ satisfying (12) is an enriched Chatterjea contraction (as introduced in [22], Definition 2.1), and so we may denote, by $\mathcal{C}_{C}^{e}(X)$, the set of all self mappings of $X$ satisfying (12).

Obviously, any Chatterjea mapping satisfies (12) with $k=0$; that is, $\mathcal{C}_{C}(X) \subseteq \mathcal{C}_{C}^{e}(X)$.

However, as shown by the following example, the inclusion $\mathcal{C}_{C}(X) \subset \mathcal{C}_{C}^{e}(X)$ is strict, as not every enriched Chatterjea contraction is a Chatterjea contraction.

Example 3. Let $X=[0,1]$ be endowed with the usual norm and $T: X \rightarrow X$ be defined by $T x=1-x$, for all $x \in[0,1]$. We first prove that $T \in \mathcal{C}_{C}^{e}(X)$. Indeed, the enriched Chatterjea condition (12) is in this case equivalent to

$$
|(k-1)(x-y)| \leq b[|(k+1) x-(k-1) y-1|+|(k+1) y-(k-1) x-1|] .
$$

As

$$
\begin{gathered}
2 k|x-y|=|[(k+1) x-(k-1) y-1]-[(k+1) y-(k-1) x-1]| \\
\leq|(k+1) x-(k-1) y-1|+|(k+1) y-(k-1) x-1|,
\end{gathered}
$$

then in order to have (13) satisfied for all $x, y \in[0,1]$, it is necessary to have $\frac{|k-1|}{2 k} \leq b$, for a certain $b \in[0,1 / 2)$. 
The only possibility is having $k<1$ when, by taking $b=\frac{1-k}{2 k}$, we obtain $k=\frac{1}{b+2}$.

Therefore, for any $b \in[0,1 / 2)$ and $\lambda=\frac{b+2}{b+3}$, we have $T_{\lambda} \in \mathcal{C}_{C}(X)$.

But $T \notin \mathcal{C}_{C}(X)$. Indeed, if $T$ is a Chatterjea mapping, then, in view of (4), there would be $b \in[0,1 / 2)$, such that, for all $x, y \in[0,1]$,

$$
|x-y| \leq 2 b \cdot|x+y-1|,
$$

which, for $x=0$ and $y=1$, yields the contradiction $1 \leq 0$.

So, $T \in \mathcal{C}_{C}^{e}(X)$ but $T \notin \mathcal{C}_{C}(X)$.

Therefore, the above facts enable us to formulate the following result.

Proposition 3. Let $(X,\|\cdot\|)$ be a Banach space. Then, $\mathcal{C}_{C}(X)$ is an unsaturated class of contractive mappings.

Hence, Proposition 3 guaranties that the next theorem, which is the main result in [22], is a genuine generalization of the Chatterjea's fixed point theorem in the setting of Banach spaces.

Theorem 5 ([22], Theorem 2.1). Let $(X,\|\cdot\|)$ be a Banach space and $T: X \rightarrow X a(k, b)$-enriched Chatterjea mapping. Then

(i) Fix $(T)=\{p\}$, for some $p \in X$;

(ii) There exists $\lambda \in(0,1]$ such that the iterative method $\left\{x_{n}\right\}_{n=0}^{\infty}$, given by

$$
x_{n+1}=(1-\lambda) x_{n}+\lambda T x_{n}, n \geq 0,
$$

converges to $p$, for any $x_{0} \in X$;

(iii) The following estimate holds

$$
\left\|x_{n+i-1}-p\right\| \leq \frac{\delta^{i}}{1-\delta} \cdot\left\|x_{n}-x_{n-1}\right\|, \quad n=0,1,2, \ldots ; i=1,2, \ldots
$$

where $\delta=\frac{b}{1-b}$.

\subsection{Almost Contractions}

Let $(X, d)$ be a metric space and let $\mathcal{C}_{A C}(X)$ denote the class of almost contractions defined on $X$, that is, the class of all mappings which satisfy a contractive condition of the form (5) on $X$.

Consider the set of all mappings $T: X \rightarrow X$ which satisfy:

$$
\|b(x-y)+T x-T y\| \leq \theta\|x-y\|+L\|b(x-y)+T x-y\|, \forall x, y \in X,
$$

for some constants $b \in[0, \infty), \theta \in(0, b+1)$ and $L \geq 0$, where $(X,\|\cdot\|)$ is a linear normed space.

If we put $\lambda=\frac{1}{b+1}$, then $0<\lambda \leq 1$, and from (14) we obtain that

$$
\left\|T_{\lambda}(x)-T_{\lambda} y\right\| \leq \delta\|x-y\|+L\left\|T_{\lambda} x-y\right\|, \forall x, y \in X,
$$

with $\delta=\frac{\theta}{b+1} \in(0,1)$, which shows that $T_{\lambda} \in \mathcal{C}_{A C}(X)$.

According to Definition 2, this means that $T$ satisfying (14) is an enriched almost contraction (as introduced in [23], Definition 3). Therefore, we denote, by $\mathcal{C}_{A C}^{e}(X)$, the set of all self mappings of $X$ satisfying (14).

Obviously, any almost contraction satisfies (14) with $b=0$, that is, $\mathcal{C}_{A C}(X) \subseteq \mathcal{C}_{A C}^{e}(X)$. 
However, as shown by the next example, the inclusion $\mathcal{C}_{A C}(X) \subset \mathcal{C}_{A C}^{e}(X)$ is strict.

Example 4 ([23], Example 3). Let $X=\left[0, \frac{4}{3}\right]$ with the usual norm and $T: X \rightarrow X$ be given by

$$
T x=\left\{\begin{array}{l}
1-x, \text { if } x \in\left[0, \frac{2}{3}\right) \\
2-x, \text { if } x \in\left[\frac{2}{3}, \frac{4}{3}\right]
\end{array}\right.
$$

Then-see Example 3 in [23] for details- $T_{\lambda} \in \mathcal{C}_{A C}(X)$, and hence $T \in \mathcal{C}_{A C}^{e}(X)$, but $T \notin \mathcal{C}_{A C}(X)$ (just let $x=\frac{7}{15}$ and $y=\frac{8}{15}$ in the almost contraction condition (5) to obtain

$$
\frac{1}{15} \leq \delta \cdot \frac{1}{15}<\frac{1}{15}
$$

a contradiction).

Note that in this case Fix $(T)=\left\{\frac{1}{2}, 1\right\}$.

Now, the above facts enable us to formulate the following result.

Proposition 4. Let $(X,\|\cdot, \cdot\|)$ be a Banach space. Then, $\mathcal{C}_{A C}(X)$ is an unsaturated class of contractive mappings.

Therefore, Proposition 4 guarantees that the next theorem, which is the main result in [23], is a genuine generalization of Theorem 1 [9], in the setting of Banach spaces.

Theorem $6([9]$, Theorem 1$)$. Let $(X,\|\cdot\|)$ be a Banach space and let $T: X \rightarrow X$ be $a(b, \theta, L)$ almost contraction. Then

(1) $\operatorname{Fix}(T) \neq \varnothing$;

(2) For any $x_{0} \in X$, there exists $\lambda \in(0,1)$ such that the Krasnoselkij iteration $\left\{x_{n}\right\}_{n=0}^{\infty}$ defined by

$$
x_{n+1}=(1-\lambda) x_{n}+\lambda T x_{n}, n \geq 0,
$$

converges to some $x^{*} \in$ Fix $(T)$, for any $x_{0} \in X$;

(3) The following estimate holds

$$
\left\|x_{n+i-1}-x^{*}\right\| \leq \frac{\delta^{i}}{1-\delta}\left\|x_{n}-x_{n-1}\right\|, \quad n=0,1,2, \ldots ; i=1,2, \ldots,
$$

where $\delta=\frac{\theta}{b+1}$.

Remark 2. We note that Theorem 6 unifies Theorems 1-5, and many other important fixed-point theorems in metrical fixed-point theory.

\subsection{Nonexpansive Mappings}

Let $(X,\|\cdot\|)$ be a linear normed space and let $\mathcal{C}_{N E}(X)$ denote the class of nonexpansive self mappings defined on $X$; that is, the class of all mappings which satisfy the contractive condition

$$
\|T x-T y\| \leq\|x-y\|, \text { for all } x, y \in X .
$$

Consider the set of all mappings $T: X \rightarrow X$ which satisfy:

$$
\|b(x-y)+T x-T y\| \leq(b+1)\|x-y\|, \forall x, y \in X .
$$


for some $b \in[0, \infty)$. By letting $\lambda=\frac{1}{b+1}$ in (16), we obtain that $T_{\lambda}$ satisfies (15), that is, $T_{\lambda} \in \mathcal{C}_{N E}(X)$. According to Definition 2, this means that $T$ satisfying (16) is an enriched nonexpansive mapping (as it was introduced in [18], Definition 2.1), and so we denote by $\mathcal{C}_{N E}^{e}(X)$ the set of all self mappings of $X$ satisfying (16).

Obviously, any nonexpansive mapping satisfies (16) with $b=0$, which means that $\mathcal{C}_{N E}(X) \subseteq \mathcal{C}_{N E}^{e}(X)$

But, as shown by the next example, the inclusion $\mathcal{C}_{N E}(X) \subset \mathcal{C}_{N E}^{e}(X)$ is strict.

Example 5 ([18], Example 2.1). Let $X=\left[\frac{1}{2}, 2\right]$ be endowed with the usual norm and $T: X \rightarrow X$ be defined by $T x=\frac{1}{x}$, for all $x \in\left[\frac{1}{2}, 2\right]$. Then $T \notin \mathcal{C}_{N E}(X)$ (just take $x=1$ and $y=\frac{1}{2}$ in (15) to get the contradiction $1 \leq \frac{1}{2}$ ) but $T_{\lambda} \in \mathcal{C}_{N E}(X)$, see Example 2.1 in [18] for details, which shows that $T \in \mathcal{C}_{N E}^{e}(X)$.

Now, all the above facts enable us to formulate the following result.

Proposition 5. Let $H$ be a Hilbert space. Then $\mathcal{C}_{N E}(H)$ is an unsaturated class of contractive mappings.

Proposition 5 now guaranties that the next theorem, which is the main result in [18], is a genuine generalization of Lemma 3 of Petryshyn [31] and of its global variant (Theorem 6) in Browder and Petryshyn [26], in the setting of Hilbert spaces.

Theorem 7 ([18], Theorem 2.2). Let $C$ be a bounded closed convex subset of a Hilbert space $H$ and $T: C \rightarrow C$ be a b-enriched nonexpansive and demicompact mapping. Then, the set Fix $(T)$ of fixed points of $T$ is a nonempty convex set and there exists $\lambda \in(0,1)$ such that, for any given $x_{0} \in C$, the Krasnoselskij iteration $\left\{x_{n}\right\}_{n=0}^{\infty}$ given by

$$
x_{n+1}=(1-\lambda) x_{n}+\lambda T x_{n}, n \geq 0,
$$

converges strongly to a fixed point of $T$.

Remark 3. The corresponding result to Theorem 7 in the setting of Banach spaces has been established in [19].

\section{Saturated Classes of Contractive Mappings}

According to Definition 3, the class of enriched mappings with respect to a certain class $\mathcal{C}$ of mappings is saturated.

As a direct consequence, the classes of enriched mappings presented in the previous section, i.e., the class of enriched Banach contractions, $\mathcal{C}_{B}^{e}(X)$, the class of enriched Kannan contractions, $\mathcal{C}_{K}^{e}(X)$, the class of enriched Chatterjea contractions, $\mathcal{C}_{C}^{e}(X)$, the class of enriched almost contractions, $\mathcal{C}_{A C}^{e}(X)$, and the class of enriched nonexpansive mappings, $\mathcal{C}_{N E}^{e}(X)$, are all examples of saturated classes of contractive mappings.

No significant new fixed-point result can be obtained by applying the technique of enrichment to any of these mapping classes.

In the following, we analyse two other important classes of contractive mappings in the metric fixed-point theory: that of the strictly pseudo-contractive mappings and that of the demicontractive mappings. We aim to show that these are also saturated classes of contractive mappings. 


\subsection{Strictly Pseudo-Contractive Mappings}

Let $C$ be a nonempty subset of a normed space $X$. A mapping $T: C \rightarrow C$ is called $k$-strictly pseudocontractive [26] if there exists $k \in(0,1)$ such that

$$
\|T x-T y\|^{2} \leq\|x-y\|^{2}+k\|x-y-(T x-T y)\|^{2}, \forall x, y \in C .
$$

If (17) holds with $k=1$, then $T$ is called pseudocontractive.

Obviously, any strictly pseudocontractive mapping is pseudocontractive, but the converse is not generally true.

There is a rich literature devoted to the study of (strictly) pseudocontractive mappings. For example, by searching in MathScinet, we found 134 publications including the term "strictly pseudocontractive" in their title, while 229 publications included the term "strictly pseudocontractive" in the review text or in their title.

In the following, we shall uuse $\mathcal{C}_{S P C}(X)$ to denote the class of strictly pseudocontractive mappings on $X$.

The next example shows that, while any nonexpansive mapping is strictly pseudocontractive, the reverse is not true.

Example 6. Let $X$ and $T$, as in Example 5. In this case, Condition (17) becomes

$$
\left|\frac{1}{x}-\frac{1}{y}\right|^{2} \leq|x-y|^{2}+k \cdot\left|x-y-\left(\frac{1}{x}-\frac{1}{y}\right)\right|^{2}
$$

and it holds for all $x, y \in\left[\frac{1}{2}, 2\right]$ if $k \in\left(\frac{3}{5}, 1\right)$.

Hence, $T \notin \mathcal{C}_{N E}(X)$ (just take $x=1$ and $y=\frac{1}{2}$ in (15) to reach to the contradiction $1 \leq \frac{1}{2}$ ), but $T \in \mathcal{C}_{S P C}(X)$.

Example 6 shows that $\mathcal{C}_{N E}(X)$ is strictly included in $\mathcal{C}_{S P C}(X)$. This suggests that we should check whether or not $\mathcal{C}_{S P C}(X)=\mathcal{C}_{N E}^{e}(X)$.

Theorem 8. Let $H$ be a real Hilbert space. Then, $\mathcal{C}_{S P C}(H)$ is a saturated class of contractive mappings.

Proof. It is enough to show that $\mathcal{C}_{S P C}(H)=\mathcal{C}_{N E}^{e}(H)$. Let $T \in \mathcal{C}_{S P C}(H)$. Then $T$ satisfies (17) with $k \in(0,1)$. But

$$
\begin{gathered}
\|x-y-(T x-T y)\|^{2}=\langle x-y-(T x-T y), x-y-(T x-T y)\rangle \\
=\|x-y\|^{2}-2\langle x-y, T x-T y\rangle+\|T x-T y\|^{2}
\end{gathered}
$$

and so (17) is equivalent to

$$
\|T x-T y\|^{2} \leq \frac{1+k}{1-k} \cdot\|x-y\|^{2}-\frac{2 k}{1-k} \cdot\langle x-y, T x-T y\rangle .
$$

By adding both sides of the previous inequality, the quantity

$$
\left(\frac{k}{1-k}\right)^{2} \cdot\|x-y\|^{2}+\frac{2 k}{1-k}\langle x-y, T x-T y\rangle,
$$

we deduce that (17) is equivalent to

$$
\left\|\frac{k}{1-k}(x-y)+T x-T y\right\|^{2} \leq\left(\left(\frac{k}{1-k}\right)^{2}+\frac{1+k}{1-k}\right)\|x-y\|^{2} .
$$


Now, by denoting $b=\frac{k}{1-k}>0$, it follows that the inequality (18) is equivalent to

$$
\|b(x-y)+T x-T y\| \leq(b+1)\|x-y\|, \forall x, y \in C,
$$

which shows that $T \in \mathcal{C}_{N E}^{e}(H)$.

The converse follows by the fact that all transformations above are equivalent.

Remark 4. An indirect consequence of Theorem 8 is that Theorem 2.1 in [17], which gives a strong convergence theorem for Krasnoselskij iteration in the class of strictly pseudocontractive mappings in Hilbert spaces is not an effective extension of Theorem 12 in Browder and Petryshyn [26].

However, in the case of a Banach space $X$, the class of enriched nonexpansive mappings, $\mathcal{C}_{N E}^{e}(X)$ does not coincide with the class of strictly pseudo-contractive mappings $\mathcal{C}_{S P C}(X)$. Therefore, Theorem 3.2 in [19] is a genuine generalization of Theorem 12 in Browder and Petryshyn [26] and also of Theorem 1 in Senter and Dotson [32], which, in turn, is an extension of Theorem 12 in Browder and Petryshyn [26].

\subsection{Demicontractive Mappings}

Let $C$ be a nonempty subset of a normed space $X$. A mapping $T: C \rightarrow C$ is called $k$-demicontractive if $\operatorname{Fix}(T) \neq \varnothing$ and there exists $k \in(0,1)$ such that

$$
\left\|T x-y^{*}\right\|^{2} \leq\left\|x-y^{*}\right\|^{2}+k\|x-T x\|^{2}, \forall x \in C, \forall y^{*} \in \operatorname{Fix}(T) .
$$

Recall that $T$ is said to be quasi-nonexpansive if $\operatorname{Fix}(T) \neq \varnothing$ and

$$
\left\|T x-y^{*}\right\| \leq\left\|x-y^{*}\right\|, \forall x \in C, \forall y^{*} \in \operatorname{Fix}(T) .
$$

It is easy to see that every quasi-nonexpansive mapping and every nonexpansive mapping with $\operatorname{Fix}(T) \neq \varnothing$ are demicontractive, but the reverse is not valid.

Note also that if we take $y=y^{*} \in$ Fix (T) in (17), we obtain the exact condition (19). This shows that any strictly pseudocontractive mapping is also demicontractive but the reverse is not generally true. The class of demicontractive mappings was introduced and studied independently in 1977 by Măruşter [33], see also [34], and Hicks and Kubicek [35], see Chidume and Măruşter [36].

Denote, using $\mathcal{C}_{D C}(X)$ and $\mathcal{C}_{Q N E}(X)$, the classes of demicontractive mappings and quasi-nonexpansive mappings on $X$, respectively.

Example 7 ([36]). Let $H$ be the real line and $C=[0,1]$. Define $T$ on $C$ by $T x=\frac{2}{3} x \sin \frac{1}{x}$, if $x \neq 0$ and $T 0=0$. Then, Fix $(T)=\{0\}$. For any $x \in C$ and $y^{*}=0$, we have

$$
|T x-0|^{2}=|T x|^{2}=\left|\frac{2}{3} x \sin (1 / x)\right|^{2} \leq\left|\frac{2}{3} x\right|^{2} \leq|x|^{2} \leq|x-0|^{2}+k|T x-x|^{2},
$$

which holds for any $k<1$. Hence (19) is satisfied, so $T$ is demicontractive (and also quasinonexpansive).

However, $T$ is not nonexpansive. Just take $x=\frac{2}{\pi}$ and $y=\frac{2}{3 \pi}$ to get

$$
|T x-T y|=\frac{16}{9 \pi}>\frac{4}{3 \pi}=|x-y| .
$$

Note also that $T$ is not pseudocontractive, and hence is not strictly pseudocontractive either. To observe this, we take the same values $x=\frac{2}{\pi}$ and $y=\frac{2}{3 \pi}$ as above to get

$$
|T x-T y|^{2}=\frac{256}{81 \pi^{2}}>\frac{160}{81 \pi^{2}}=|x-y|^{2}+|(x-y)-(T x-T y)|^{2} .
$$


There exists a rich literature devoted to the study of demicontractive mappings. For example, by searching in MathScinet, we find 108 publications including the term "demicontractive" in their title, while 152 publications include the term "demicontractive" in the review text or title.

We now state the main result of this subsection.

Theorem 9. Let $H$ be a real Hilbert space. Then, $\mathcal{C}_{D C}(H)$ is a saturated class of contractive mappings.

Proof. It is enough to show that $\mathcal{C}_{D C}(H)$ coincides with the class of enriched quasi nonexpansive mappings, $\mathcal{C}_{\mathrm{QNE}}^{e}(H)$.

In accorance with the definition of enriched nonexpansive mappings, it follows that a mapping $T$ is enriched quasi-nonexpansive if there exists $b \in[0, \infty)$ such that

$$
\left\|b\left(x-y^{*}\right)+T x-y^{*}\right\| \leq(b+1)\left\|x-y^{*}\right\|, \forall x \in X \text { and } y^{*} \in \operatorname{Fix}(T) .
$$

Let $T \in \mathcal{C}_{D C}(H)$. This means that $T$ satisfies (19) with $k \in(0,1)$, that is,

$$
\left\|T x-y^{*}\right\|^{2} \leq\left\|x-y^{*}\right\|^{2}+k\|x-T x\|^{2}, \forall x \in X \text { and } y^{*} \in \operatorname{Fix}(T) .
$$

Similarly to the proof of Theorem 8 , we have

$$
\begin{gathered}
\|x-T x\|^{2}=\left\|x-y^{*}-\left(T x-y^{*}\right)\right\|^{2}=\left\langle x-y^{*}-\left(T x-y^{*}\right), x-y^{*}-\left(T x-y^{*}\right)\right\rangle \\
=\left\|x-y^{*}\right\|^{2}-2\left\langle x-y^{*}, T x-y^{*}\right\rangle+\left\|T x-y^{*}\right\|^{2}
\end{gathered}
$$

and so (19) is equivalent to

$$
\left\|T x-y^{*}\right\|^{2} \leq \frac{1+k}{1-k} \cdot\left\|x-y^{*}\right\|^{2}-\frac{2 k}{1-k} \cdot\left\langle x-y^{*}, T x-y^{*}\right\rangle .
$$

Now, by adding both sides of the previous inequalit,y the quantity

$$
\left(\frac{k}{1-k}\right)^{2} \cdot\left\|x-y^{*}\right\|^{2}+\frac{2 k}{1-k}\left\langle x-y^{*}, T x-y^{*}\right\rangle,
$$

we obtain the equivalent inequality

$$
\left\|\frac{k}{1-k}\left(x-y^{*}\right)+T x-y^{*}\right\|^{2} \leq\left(\left(\frac{k}{1-k}\right)^{2}+\frac{1+k}{1-k}\right)\left\|x-y^{*}\right\|^{2} .
$$

Therefore, by denoting $b=\frac{k}{1-k}$, it follows that the inequality (22) is equivalent to

$$
\left\|b\left(x-y^{*}\right)+T x-y^{*}\right\| \leq(b+1)\left\|x-y^{*}\right\|, \forall x, y \in C,
$$

which shows that $T \in \mathcal{C}_{O N E}^{e}(H)$.

The converse follows through the fact that all the above transformations are equivalent.

Remark 5. Theorem 9 shows that the class of demicontractive mappings cannot be enlarged by the technique of enriching contractive mappings.

However, there are many other important classes of contractive mappings in the metric fixed-point theory, e.g., the Ćirić-Reich-Rus contractions and Ćirić quasi contractions, that deserve to be investigated in order to establish whether they are (un)saturated or not. 
Let $(X, d)$ be a metric space. In 1971, Ćirić [37], Reich [38] and Rus [39] have established independently a fixed point theorem for mappings $T: X \rightarrow X$ satisfying the following condition

$$
d(T x, T y) \leq a d(x, y)+b(d(x, T x)+d(y, T y)), \text { for all } x, y \in X,
$$

where $a, b \geq 0$ and $a+2 b<1$. The coefficients $a, b$ may depend on $x, y$, by adding an appropriate additional boundedness condition; see Ćirić [37] and Reich [40].

Denote, using $\mathcal{C}_{C R R}(X)$, the class of Ćirić-Reich-Rus contractions on $X$, that is, the class of mappings which satisfy the contractive condition (23) on the metric space $(X, d)$.

We note that if $b=0$, condition (23) reduces to Banach's contraction condition (1), while, for $a=0$ condition (23) reduces to Kannan's contraction condition (3).

Open problem 1. Let $X$ be a Banach space. Is $\mathcal{C}_{C R R}(X)$ a saturated/unsaturated class of contractive mappings?

Denote by $\mathcal{C}_{C Q}(X)$ the class of Ćirić quasi-contractions on $X$, that is, of mappings which satisfy the contractive condition (6) on the metric space $(X, d)$.

Open problem 2. Let $X$ be a Banach space. Is $\mathcal{C}_{C Q}(X)$ a saturated/unsaturated class of contractive mappings?

Similar open problems could be formulated for the class of F-contractions, due to Wardowski [41], and for the very diversified classes of mappings in the family of nonexpansive mappings: asymptotically nonexpansive mappings; asymptotically quasi-nonexpansive mappings; $\alpha$-nonexpansive mappings; Suzuki nonexpansive mappings; Suzuki generalized nonexpansive mappings; firmly nonexpansive mappings; Bregman nonexpansive; affine nonexpansive; nearly nonexpansive mappings; G-nonexpansive mappings; iterated nonexpansive mappings, etc.

\section{Conclusions and Further Study}

1. Based on the technique of enriching contractive type mappings $T$ by means of the averaged operator $T_{\lambda}$, we introduced the concept of saturated class of contractive mappings;

2. We have shown that, from this perspective, the contractive type mappings in metric fixed-point theory could be separated into two distinct classes, unsaturated contractive mappings and saturated contractive mappings;

3. We identified some important unsaturated mapping classes by surveying some significant fixed-point results reported recently for five remarkable classes of contractive mappings. We have shown that the technique of enriching contractive type mappings provided genuine new fixed-point results for all mappings;

4. In the second part of the paper, we also identified two important saturated classes of contractive mappings: the class of strictly pseudocontractive mappings and the class of demicontractive mappings. The essential feature of these saturated classes of mappings is that they cannot be expanded by means of the technique of enriching contractive mappings;

5. We also formulated two open problems, which are intended to establish whether Ćirić-Reich-Rus contractions and Ćirić quasi contractions are saturated or unsaturated contraction classes;

6. In a further study, we plan to consider the same problem as above for other contractive type mappings from metric fixed-point theory, shown in in $[7,8,10,15,16,42-53]$ and the references therein.

Author Contributions: Both authors contributed equally to this work. Both authors have read and agreed to the published version of the manuscript. 
Funding: The first author has been financially supported by Department of Mathematics, Faculty of Sciences, North University Centre at Baia Mare, Technical University of Cluj-Napoca.

Acknowledgments: The first author acknowledges the constant support offered by the Department of Mathematics, Faculty of Sciences, North University Centre at Baia Mare, Technical University of Cluj-Napoca.

Conflicts of Interest: The authors declare no conflict of interest.

\section{References}

1. Rus, I.A. Generalized Contractions and Applications; Cluj University Press: Cluj-Napoca, Romania, 2001.

2. Banach, S. Sur les opérations dans les ensembles abstraits et leurs applications aux équations intégrales. Fundam. Math. 1922, 3, 133-181. [CrossRef]

3. Caccioppoli, R. Un teorema generale sull'esistenza di elementi uniti in una transformazione funzionale. Rend. Accad. Lincei 1930, 11, 794-799.

4. Kannan, R. Some results on fixed points. Bull. Calcutta Math. Soc. 1968, 60, 71-76.

5. Kannan, R. Some results on fixed points. II. Am. Math. Mon. 1969, 76, 405-408.

6. Chatterjea, S.K. Fixed-point theorems. C. R. Acad. Bulg. Sci. 1972, 25, 727-730. [CrossRef]

7. Meszaros, J. A comparison of various definitions of contractive type mappings. Bull. Calcutta Math. Soc. 1992, 84, 167-194.

8. Rhoades, B.E. A comparison of various definitions of contractive mappings. Trans. Am. Math. Soc. 1977, 226, 257-290. [CrossRef]

9. Berinde, V. Approximating fixed points of weak contractions using the Picard iteration. Nonlinear Anal. Forum 2004, 9, 43-53.

10. Berinde, V. Iterative Approximation of Fixed Points, 2nd ed.; Lecture Notes in Mathematics, 1912; Springer: Berlin, Germany, 2007.

11. Păcurar, M. Iterative Methods for Fixed Point Approximation; Editura Risoprint: Cluj-Napoca, Romania, 2009.

12. Zamfirescu, T. Fix point theorems in metric spaces. Arch. Math. 1972, 23, 292-298. [CrossRef]

13. Berinde, V.; Păcurar, M. Iterative approximation of fixed points of single-valued almost contractions. In Fixed Point Theory and Graph Theory; Elsevier/Academic Press: Amsterdam, The Netherlands, 2016; pp. 29-97.

14. Ćirić, L.B. A generalization of Banach's contraction principle. Proc. Am. Math. Soc. 1974, 45, 267-273. [CrossRef]

15. Rus, I.A. Principles and Applications of the Fixed Point Theory; Editura Dacia: Cluj-Napoca, Romania, 1979. (In Romanian)

16. Rus, I.A.; Petruşel A.; Petruşel, G. Fixed Point Theory; Cluj University Press: Cluj-Napoca, Romania, 2008.

17. Berinde, V. Weak and strong convergence theorems for the Krasnoselskij iterative algorithm in the class of enriched strictly pseudocontractive operators. An. Univ. Vest Timiş. Ser. Mat.-Inform. 2018, 56, 13-27. [CrossRef]

18. Berinde, V. Approximating fixed points of enriched nonexpansive mappings by Krasnoselskij iteration in Hilbert spaces. Carpathian J. Math. 2019, 35, 293-304. [CrossRef]

19. Berinde, V. Approximating fixed points of enriched nonexpansive mappings in Banach spaces by using a retraction-displacement condition. Carpathian J. Math. 2020, 36, 27-34. [CrossRef]

20. Berinde, V.; Păcurar, M. Approximating fixed points of enriched contractions in Banach spaces. J. Fixed Point Theory Appl. 2020, 22, 1-10. [CrossRef]

21. Berinde, V.; Păcurar, M. Kannan's fixed point approximation for solving split feasibility and variational inequality problems. J. Comput. Appl. Math. 2021, 386, 113217. [CrossRef]

22. Berinde, V.; Păcurar, M. Fixed point theorems for Chatterjea type mappings in Banach spaces. J. Fixed Point Theory Appl. 2021. under review.

23. Berinde, V.; Păcurar, M. Krasnoselskij-type algorithms for variational inequality problems and fixed point problems in Banach spaces. arXiv 2021, arXiv:2103.10289.

24. Browder, F.E.; Petryshyn, W.V. The solution by iteration of nonlinear functional equations in Banach spaces. Bull. Amer. Math. Soc. 1966, 72, 571-575. [CrossRef]

25. Krasnosel'skii, M.A. Two remarks about the method of successive approximations. Uspehi Mat. Nauk (N.S.) 1955, 10, 123-127. (In Russian)

26. Browder, F.E.; Petryshyn, W.V. Construction of fixed points of nonlinear mappings in Hilbert space. J. Math. Anal. Appl. 1967, 20, 197-228. [CrossRef]

27. Ishikawa, S. Fixed point and iteration of a non-expansive mapping in a Banach space. Proc. Am. Math. Soc. 1976, 59, 65-71. [CrossRef]

28. Kesahorm, T.; Sintunavarat, W. On novel common fixed point results for enriched nonexpansive semigroups. Thai J. Math. 2020, $18,1549-1563$.

29. Takahashi, W. A convexity in metric space and nonexpansive mappings. I. Kōdai Math. Sem. Rep. 1970, 22, 142-149. [CrossRef]

30. Baillon, J.B.; Bruck R.E.; Reich, S. On the asymptotic behavior of nonexpansive mappings and semigroups in Banach spaces. Houst. J. Math. 1978, 4, 1-9.

31. Petryshyn, W.V. Construction of fixed points of demicompact mappings in Hilbert space. J. Math. Anal. Appl. 1966, 14, 276-284. [CrossRef]

32. Senter, H.F.; Dotson, W.G., Jr. Approximating fixed points of nonexpansive mappings. Proc. Am. Math. Soc. 1974, 44, 375-380. [CrossRef] 
33. Măruşter, Ş. The solution by iteration of nonlinear equations in Hilbert spaces. Proc. Am. Math. Soc. 1977, 63, 69-73. [CrossRef]

34. Măruşter, S. Sur le calcul des zéros d'un opérateur discontinu par itération. Canad. Math. Bull. 1973, 16, 541-544. [CrossRef]

35. Hicks, T.L.; Kubicek, J.D. On the Mann iteration process in Hilbert spaces. J. Math. Anal. Appl. 1977, 59, 498-504. [CrossRef]

36. Chidume, C.E.; Măruşter, Ş. Iterative methods for the computation of fixed points of demicontractive mappings. J. Comput. Appl. Math. 2010, 234, 861-882. [CrossRef]

37. Ćirić, L.B. Generalized contractions and fixed-point theorems. Publ. Inst. Math. (Beogr.) (N.S.) 1971, 12, $19-26$.

38. Reich, S. Some remarks concerning contraction mappings. Canad. Math. Bull. 1971, 14, 121-124. [CrossRef]

39. Rus, I.A. Some fixed point theorems in metric spaces. Rend. Istit. Mat. Univ. Trieste 1971, 3, 169-172.

40. Reich, S. Fixed points of contractive functions. Boll. Un. Mat. Ital. 1972, 5, 26-42.

41. Wardowski, D. Fixed points of a new type of contractive mappings in complete metric spaces. Fixed Point Theory Appl. 2012, 2012, 1-6. [CrossRef]

42. Alghamdi, M.; Berinde, V.; Shahzad, N. Fixed point of multivalued nonself almost contractions. J. Appl. Math. 2013. [CrossRef]

43. Berinde, V. Approximating fixed points of implicit almost contractions. Hacettepe J. Math. Stat. 2012, 41, 93-102.

44. Berinde, V.; Păcurar, M. Fixed point theorems for nonself single-valued almost contractions. Fixed Point Theory 2013, 14, 301-311.

45. Berinde, V.; Rus, I.A. Asymptotic regularity, fixed points and successive approximations. Filomat 2020, 34, 965-981. [CrossRef]

46. Reich, S.; Shafrir, I. Nonexpansive iterations in hyperbolic spaces. Nonlinear Anal. 1990, 15, 537-558. [CrossRef]

47. Reich, S.; Salinas, Z. Weak convergence of infinite products of operators in Hadamard spaces. Rend. Circ. Mat. Palermo 2016, 65, 55-71. [CrossRef]

48. Reich, S. ; Zaslavski, A.J. Attracting mappings in Banach and hyperbolic spaces. J. Math. Anal. Appl. 2001, 253, 250-268. [CrossRef]

49. Reich, S. ; Zaslavski, A.J. Two porosity theorems for nonexpansive mappings in hyperbolic spaces. J. Math. Anal. Appl. 2016, 433, 1220-1229. [CrossRef]

50. Rus, I.A. Metrical Fixed Point Theorems; Univ. of Cluj-Napoca: Cluj-Napoca, Romania, 1979.

51. Rus, I.A. Generalized contractions. Semin. Fixed Point Theory 1983, $31-130$.

52. Rus, I.A. Picard operators and applications. Sci. Math. Jpn. 2003, 58, 191-219.

53. Rus, I.A. Heuristic introduction to weakly Picard operator theory. Creat. Math. Inform. 2014, 23, $243-252$. 